\title{
Module's Robust Design Based on Product Interface Association Model
}

\author{
Jia Haili ${ }^{1+}$, Zhou Zhengdao ${ }^{1}$ and Meng Xiangjie ${ }^{2}$ \\ ${ }^{1}$ School of Mechanical Engineering, Tianjin University of Technology and Education, Tianjin 300222 \\ ${ }^{2}$ Tianjin EGTree Electromechanical Equipment Co., Ltd. Tianjin 300350
}

\begin{abstract}
Design conflicts reduced by complex parameter change influence propagation require repeated cooperation in product modular design. Aiming at this problem, a method of robust modular design based on product interface association constraint network model is put forward. Interface association model which has fined to parameter level is created. Second, through analysis a kind of search arithmetic for parameter change propagation path is put forward. Third, robust design ideal is brought into modular design to establish calculation formula of robust degree and robustness evaluation standard taking module influenced degre. Finally, correctness of this technology are validated taking the engine series products as the example.
\end{abstract}

Keywords: product family, robust modular design, interface association model, influence propagation analysis of parameters change

\section{Introduction}

Modular design is to establish product function module system and to obtain modular product relationships utilizing modular principles and methods. Researches on the modular design technology are paid more attention to no matter abroad or in the domestic. There are many literatures about modular research but it also needs to study further more. Reasons are as follows.

1) in aspect of robust design:

Leandro F.F. Miguel [1] presented a novel application of robust design optimization of TMD to vehiclebridge coupled vibration problems. Cao Ming-Jie [2] proposes a robust design method for improving the stability of optimized structures. Anders Forslund [3] studied how a robust optimization scheme using genetic algorithms can improve product robustness to form error. Shortage: Currently researches of robust design mainly focus on systems' performance and function modeling, analysis, evaluation and optimization. There is no correlative research on robust design in modular design.

2) in aspect of module partition:

Janakiraman Nithiyanantham [4] proposes a novel architecture for module partitioning problems in the process of dynamic and partial reconfigurable computing. Ning Xin [5] finished the module partitioning of fractionated spacecraft on the basis of analyzing the fuzzy matter-element. Shortage: Module's independence and degree of cohesiveness are analyzed from the function point of view not considered robustness. Influence of parameters change to module and analysis of module robustness actually is important.

3) in aspect of change influence analysis:

Inayat Ullah [6] discussed the significant aspects of engineering changes, propagation paths and highlighted the methods that are proposed. Shortage: There are no systemic expression methods for

\footnotetext{
+ Corresponding author. Tel.: +15332138552; fax: +022-88181083.

E-mail address: highly0811@163.com.
} 
relationships among modules which also are not detailed to parameter lever. Aiming at above shortages, technology of product interface association modeling is studied.

\section{Interface Association Modeling of Structured Product}

Module connects with others modules by their interfaces.In structure domain, module interface is a set of association parameters which can describe orientation and figuration constrains facing a certain function unit on the component. Module robust degree means the influence degree that the change of module interface parameters act on the module itself, namely the sensitivity degree of module to the change of interface parameters. Robust modular design takes parameters change as design variable, takes module robust degree as response value, and takes getting robust module in the range of parameters change as the goal. The sensitivity of module to the change of module interface parameters can be reduced by evaluating module robustness and then improving or recombining module so that modules having robust flexibility facing all series of products can be achieved. Interface association modeling of structured product is on the basis of hierarchy structure tree of product family and uses the Top-Down design idea.

\section{Influence Analysis of Module Parameters Change}

Influence analysis of module parameters change to analyze constraint network of structured product interface association model and to calculate change propagation paths, change influence scope and change influence degree when the interface parameter of module changes. Change propagation path analysis is to obtain the set of constrain propagation paths. This set is named as the set of change propagation paths and expressed as PathSet $=\left\{P_{1}, P_{2}, \cdots, P_{i}, \cdots, P_{n}\right\} \cdot \operatorname{Path}_{i}\left\{C_{I_{i}}^{i}: p_{i}\right\}$ is as follows.

$$
\operatorname{Path}_{i}\left\{C_{I_{i}}^{i}: p_{i}\right\}=\left\{C_{I_{1}}^{1}: p_{1}, C_{I_{2}}^{2}: p_{2}, \cdots, C_{I_{i}}^{i}: p_{i}, \cdots, C_{I_{n}}^{n}: p_{n}\right\}
$$

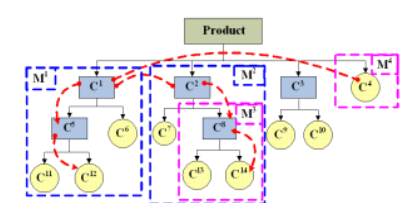

Fig. 1. Sketch map of constrain propagation paths analysis

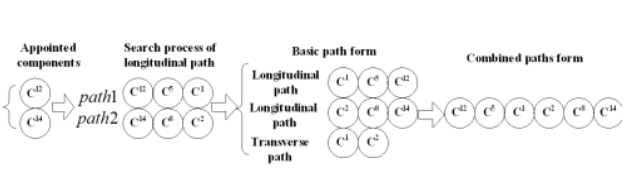

Fig. 2. Sketch map of paths search in $\mathrm{t}$ structure tree

The analysis of change propagation paths as shown in Fig.1, in product structure tree the note $C^{i}$ donates component and $M^{k}$ is module. When $I_{1}$ interface's $p_{i}$ parameter of $M^{1}$ module's $C^{5}$ component changes, the analysis process of change propagation paths are as follows.

\subsection{Path Definition}

Transverse path is composed of two components that have the same parent node in product structure tree. As shown in Fig. 1, a transverse path is composed of nodes $\left\{C^{7}, C^{8}\right\}$.

Longitudinal path is composed of these components that have father-child relationship and are connected from beginning to end in order. As shown in Fig. 1, a longitudinal transverse path is composed of nodes $\left\{C^{1}, C^{5}, C^{12}\right\}$.

Transverse-longitudinal combined path is composed of a transverse path and a longitudinal path. As shown in Fig.1, a transverse-longitudinal combined path is composed of nodes $\left\{C^{12}, C^{5}, C^{1}, C^{4}\right\}$ in which nodes $\left\{C^{1}, C^{4}\right\}$ compose a transverse path.

Longitudinal-transverse-longitudinal combined path is composed of a transverse path and two longitudinal paths. The transverse path is composed of the start nodes of the two longitudinal paths. As shown in Fig.1, a longitudinal-transverse-longitudinal combined path is composed of nodes $\left\{C^{12}, C^{5}, C^{1}, C^{2}, C^{8}, C^{14}\right\}$, while a transverse path is composed of nodes $\left\{C^{1}, C^{2}\right\}$.

\subsection{Path Search Arithmetic}

1) As shown in Fig.1, parts $C^{12}$ and $C^{14}$ are appointed as start nodes from which the structure tree is searched upwards to obtain the sequences $\left\{C^{12}, C^{5}, C^{1}\right\}$ and $\left\{C^{14}, C^{8}, C^{2}\right\}$. Then two inverse sequences is calculated to get longitudinal paths $\left\{C^{1}, C^{5}, C^{12}\right\}$ and $\left\{C^{2}, C^{8}, C^{14}\right\}$. 
2) The two top layer nodes which have the same parent node can compose a transverse path. The transverse path and the two longitudinal paths obtained in step(1) can compose a whole combined path. The process of the two longitudinal paths $\left\{C^{1}, C^{5}, C^{12}\right\}$ and $\left\{C^{2}, C^{8}, C^{14}\right\}$ (in Fig.1) and the transverse path $\left\{C^{1}, C^{2}\right\}$ composing a llongitudinal-transverse-longitudinal combined path is shown in Fig.2. According to the above method, all paths can be achieved by searching product structure tree taking any two of all parts as the starts nodes.

\subsection{Search Arithmetic of Constraint Propagation Paths}

If there is constraint propagation of parameters on the path of product structure tree, we call this path as constraint propagation path. Search methods are as follows.

1) Search Arithmetic of Transverse Constraint Propagation Paths

(1) It is to appoint two nodes $C^{i}$ and $C^{j}$ on transverse path.

(2) It is to search parameters' association relationships of $C^{i}$ and $C^{j}$ from database and to return the set of transverse constraint propagation paths $\operatorname{Constrain}_{H}=\left\{\right.$ Constrain $_{H}^{1}, \cdots$, Constrain $_{H}^{1}, \cdots$, Constrain $\left._{H}^{n}\right\}$. Constrain $_{H}^{i}=\left\{C_{I_{k}}^{i}: p_{1}, C_{I_{m}}^{j}: p_{2}\right\}$ is the $\mathrm{i}_{t h}$ transverse constraint propagation path. $p_{1}$ and $p_{2}$ are parameters of interfaces $I_{k}$ and $I_{m} . I_{k}$ and $I_{m}$ are interfaces of components $C^{i}$ and $C^{j}$. Constrain ${ }_{H}^{i}=\left\{C_{I_{k}}^{i}: p_{1}, C_{I_{m}}^{j}: p_{2}\right\}$ means there is an association constraint relationship between parameters $p_{1}$ and $p_{2}$.

2) Search Arithmetic of Longitudinal Constraint Propagation Paths

(1) It is to appoint a longitudinal path named path.

(2) It is to get two adjacent parent and child nodes $C^{m}$ and $C^{n}$. Then it is to search association relationships between $C^{m}$ and $C^{n}$ from database and return the set updown of constraint propagation paths between $C^{m}$ and $C^{n}$.

(3) It is to adjust whether we have reach the end node of path. If not then to go to step(2), else go to step (4) to calculate next node.

(4) It is to associate the constraint propagation paths between every two nodes in updown from beginning to the end in sequence of nodes on path to obtain the set of longitudinal constraint propagation paths Constrain $=\left\{\right.$ Constrain $_{v}^{1}, \cdots$, Constrain $_{v}^{i}, \cdots$, Constrain $\left._{v}^{n}\right\}$.

\subsection{Analysis Arithmetic of Change Propagation Paths}

In product structure tree as shown in Fig.1, the red curves denote the paths that contain node $C^{5}$. They are respectively the transverse-longitudinal combined path $\left\{C^{12}, C^{5}, C^{1}, C^{4}\right\}$ and the longitudinaltransverse-longitudinal combined path $\left\{C^{12}, C^{5}, C^{1}, C^{2}, C^{8}, C^{14}\right\}$. If there are constraint propagations on these two paths, the two paths respectively correspond to a transverse-longitudinal combined constraint propagation path Constrain $=\left\{C_{I_{1}}^{12}: p_{1}, C_{I_{1}}^{5}: p_{i}, C_{I_{1}}^{1}: p_{3}, C_{I_{1}}^{4}: p_{4}\right\}$ and a longitudinal-transverselongitudinal combined constraint propagation path Constrain $^{2}=\left\{C_{I_{1}}^{12}: p_{1}, C_{I_{1}}^{5}: p_{i}, C_{I_{1}}^{1}: p_{3}, C_{I_{1}}^{2}: p_{4}, C_{I_{1}}^{8}: p_{5}, C_{I_{1}}^{14}: p_{6}\right\}$. Both of the two paths contain parameter $p_{i}$, so the set of change propagation paths is PathSet $=\left\{P_{1}, P_{2}\right\}$.

$$
\begin{gathered}
\text { Path }_{1}\left\{C_{I_{1}}^{5}: p_{i}\right\}=\left\{C_{I_{1}}^{12}: p_{1}, C_{I_{1}}^{5}: p_{i}, C_{I_{1}}^{1}: p_{3}, C_{I_{1}}^{4}: p_{4}\right\} \\
\text { Path }_{2}\left\{C_{I_{1}}^{5}: p_{i}\right\}=\left\{C_{I_{1}}^{12}: p_{1}, C_{I_{1}}^{5}: p_{i}, C_{I_{1}}^{1}: p_{3}, C_{I_{1}}^{2}: p_{4}, C_{I_{1}}^{8}: p_{5}, C_{I_{1}}^{14}: p_{6}\right\} .
\end{gathered}
$$

\section{Evaluation of Module Robustness Based on Parameters' Change Influence}

Evaluation of module's robustness is to calculate the degree all parameters of module $M^{i}$ changing influence to $M^{i}$ itself based on the analysis of module parameters' changing influence. If the degree is higher the changing influence to other modules is lower and robustness of module $M^{i}$ is better. The evaluation process is as Fig.3. 


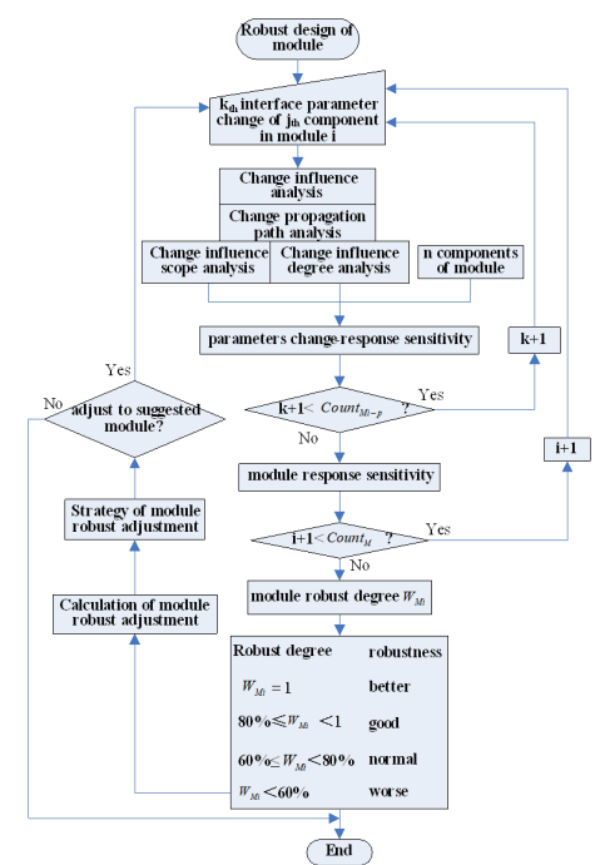

Fig. 3. Design and analysis flow of module robustness

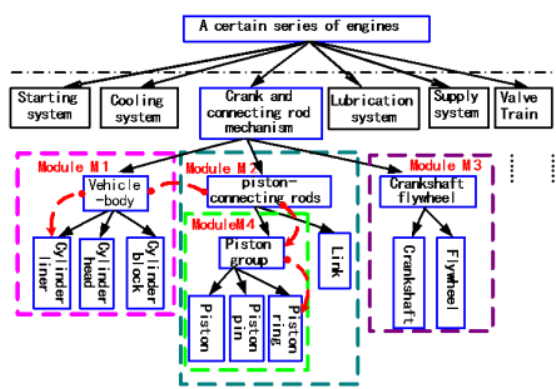

Fig. 4. The product middle structure tree of a certain series of engines

Calculation of robust degree is to appoint the module $M^{i}$ whose robustness will be judged, to acquired the amount of all interface parameters Count $_{M i-p}$, to read weight coefficient $C_{p i}$ of each interface parameter $p_{i}$ and to calculate the amount Count $_{M}$ of all modules. The calculation formula of parameters changeresponse sensitivity is as follow.

$$
X_{p i-M i}\left\{M_{I_{i}}^{i}: p_{i}, M^{i}\right\}=\sum_{i=1}^{\text {Count }_{M i-p i-C}} D_{C t}
$$

In above formula Count $_{M i-p i-C}$ is the amount of components influenced by parameter $p_{i}$ changing.

If the formula $X_{p i-M i}\left\{M_{I_{i}}^{i}: p_{i}, M^{i}\right\}=\max \left\{X_{p i-M 1}, X_{p i-M 2}, \cdots, n=\right.$ Count $_{M}$ is tenable, influence of $\left.X_{p i-M i} \cdots, X_{p i-M n}\right\}$

parameter $p_{i}$ changing to module $M^{i}$ is the highest. That is to say other modules are not sensitive to parameter $p_{i}$ changing. The calculation formula of module response sensitivity is as follow.

$$
X Z_{p-M j}\left\{M^{i}: p, M^{j}\right\}=\sum_{k=1}^{\text {Count }_{M i-p}} X_{p k-M j}
$$

In above formula, Count $_{M i-p}$ is the amount of all interface parameters in module $M^{i}$.

The formula of module's robust degree is as follow.

$$
W_{M i}=\frac{H_{M i}}{\text { Count }_{M}}
$$

In above formula $W_{M i}$ expresses the robust degree of module $M^{i}$, Count $_{M}$ is the amount of all modules and $H_{M i}$ is the sequence number.

Adjustment of components in module should be slightly adjusted according to evaluation result of robustness and should be fit for module function and structure feature. If $W_{M i}<60 \%$, module robustness is bad and optimization adjustment strategy is need to calculate.

\section{Instance Validations}

The product middle structure tree of a certain series of engines in a certain enterprise is shown in Fig.4. Interface association modeling facing series of products is shown in Fig.5. For example, when the parameter $T_{N J}$ of cylinder liner's interface $Q G T a o_{-} J K$ changes, calculation processes are done. After external 
diameter $H_{W J}$ of piston ring changes the change influence degree to cylinder liner and $M^{4}$ are shown in Fig.5. It is shown in Fig.6 that module $M^{4}$ is appointed to calculate robust degree.
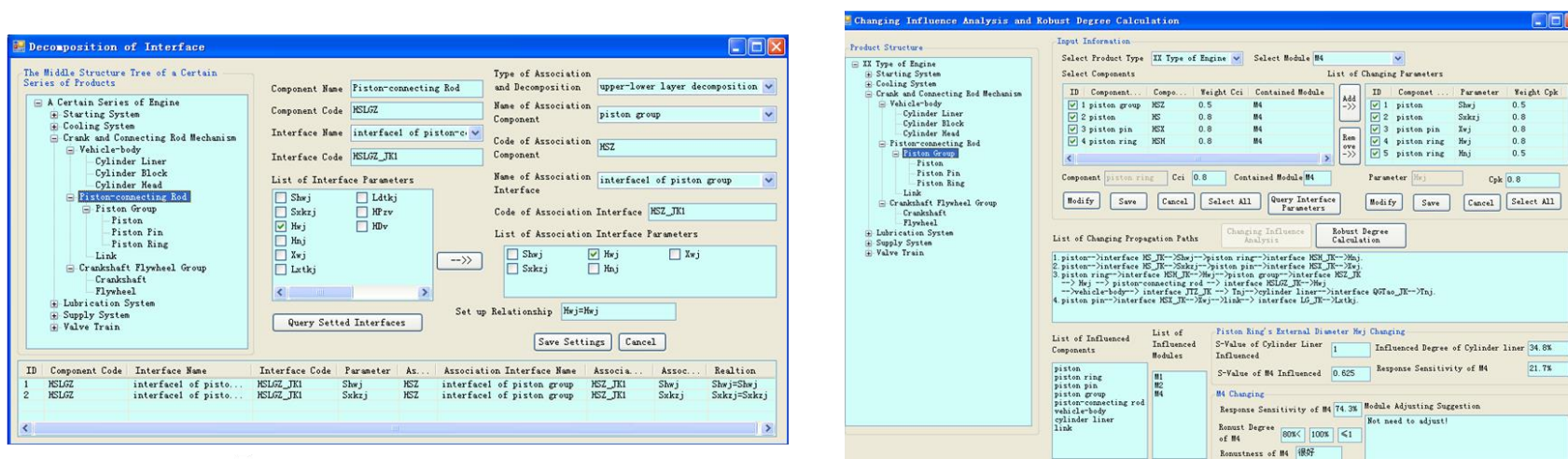

Fig. 5. Setting of interface association

Fig. 6. Analysis of changing influence and calculation of robust degree

\section{Conclusions}

Significances of this paper's contents to product modular design are summarized as follows.

1) Technology of product interface association modeling can subtly describe Top-Down structured association constraint network of product. It can reduce the constraint conflict in parameter transfer description. It also provides the model base for change influence analysis of parameters.

2) Analysis of change influence based on product interface association model provides practical tools for modular design and can promote the modular design to be practical.

3) Analysis and evaluation of module robustness provides new Ideas and methods for modular design but module robust optimization design still needs to be studied further more.

4) Product interface association modelling, change influence analysis and robust modular design are finished to validate the correctness and effectiveness of robust modular design based on product interface association model.

\section{Acknowledgements}

This paper supported by the National Natural Science Foundation of China (Grant No. 51505336), Natural Science Foundation of Tianjin (Grant No. 15JCQNJC05200), and Financing Plan for Outstanding Young Teachers of Tianjin (No. RC14-14).

\section{References}

[1] Leandro F.F. Miguel, Rafael H. Lopez, André J. Torii, etal. Robust design optimization of TMDs in vehiclebridge coupled vibration problems[J]. Engineering Structures. 2016,126(11):703-711. (in English)

[2] Cao Ming-Jie, Ma Hai-Tao, Wei Peng. A novel robust design method for improving stability of optimized structures[J]. Acta Mechanica Sinica/Lixue Xuebao. 2015,31(1): 104-111. (in English)

[3] Anders Forslund, Julia Madrid, Johan Lööf, etal. Robust Design of Aero Engine Structures: Transferring form Error Data When Mapping Out Design Spaces for New Turbine Components[J]. Procedia CIRP, 2016,43: 47-51. (in English)

[4] Janakiraman Nithiyanantham, Nirmal Kumar Palanisamy. Multi-objective module partitioning design for dynamic and partial reconfigurable system-on-chip using genetic algorithm[J]. Journal of Systems chitecture .2014,(60)1: 119-139. (in English)

[5] Ning Xin , Li Yi Fei, Yue Xiao Kui. Research on methodology of module division in fractionated spacecraft [J]. Applied Mechanics and Materials. 2013, (397-400) 943-947. (in English)

[6] Inayat Ullah, Dunbing Tang, Leilei Yin. Engineering Product and Process Design Changes: A Literature Overview[J]. Procedia CIRP, 2016, 56: 25-33. (in English) 